\title{
Distinct molecular origins for Denys-Drash and Frasier syndromes
}

\author{
F. Poulat', D. Morin ${ }^{2}$, A. König ${ }^{5}$, P. Brun ${ }^{3}$, J. Giltay ${ }^{4}$, C. Sultan' ${ }^{2}$, R. Dumas ${ }^{2}$, M. Gessler ${ }^{5}$, P. Berta \\ 1 Centre de Recherches de Biochimie Macromoléculaire, CNRS UPR 9008, INSERM U249, Route de Mende, \\ F-34033 Montpellier Cedex, France \\ ${ }^{2}$ Service de Pédiatrie I, Hôpital Saint-Charles, F-34059 Montpellier Cedex, France \\ ${ }^{3}$ Clinical Genetic Center Utrecht, P. O. Box 18009, NL-3501 CA Utrecht, The Netherlands \\ ${ }^{4}$ Service de Néphrologie, Hôpital Robert Debré, 48, Boulevard Serrurier, F-75019 Paris Cedex, France \\ 5 Institut für Humangenetik, Bahnhofstrasse 7A, W-3550 Marburg, Germany
}

Received: 23 June 1992 / Revised: 13 November 1992

\begin{abstract}
The direct involvment of the Wilm's tumor suppressor gene (WT1) in Denys-Drash syndrome through mutations within exons 8 or 9 has recently been established. The absence of such alterations in three patients with Frasier syndrome provides a molecular basis for distinguishing these two syndromes that are associated with streak gonads, pseudohermaphroditism and renal failure.
\end{abstract}

\section{Introduction}

The association of chronic renal failure with $\mathrm{XY}$ gonadal dysgenesis is well known (Drash et al. 1970; Frasier et al. 1964). However, some patients do not develop renal Wilm's tumor, but have an increased likehood of developing gonadoblastoma. These patients have been grouped under the generic term "Frasier'syndrome" by Moorthy et al. (1987). The recent discovery of the presence of point mutations in the newly described Wilm's tumor suppressor gene WT1 in 10 independent cases of Denys-Drash syndrome (Pelletier et al. 1991) has led us to search for such mutations in Frasier syndrome. We report here a sequence comparison between an individual with DenysDrash syndrome and three patients with Frasier syndrome, with respect to WT1 exons 8 and 9.

\section{Materials and methods}

The three Frasier patients (Che, Del and Mar) suffered nephrotic syndrome with important proteinuria. They all underwent renal transplantation. Their chromosome analysis revealed 46-XY with gonadal dysgenesis. Their gonads were streak gonads and, for Che, one of them was tumoral. A bilateral castration was carried out in each case.

The Denys-Drash patient (A1) had a female phenotype with XY gonadal dysgenesis and severe renal failure. Laboratory investigations confirmed the nephrotic syndrome with diffuse mesanglial sclerosis and a unilateral Wilm's tumor was discovered.

Correspondence to: P. Berta
Polymerase chain reaction (PCR) amplifications of the TW1 exons 8 or 9 were carried out using the primer sets W8A:5 -ACA GCT GCC AGC AAT GAG AA-3', W8S: $5^{\prime}-$ GAG ATC CCC AAA AGG AGT ATC A-3', and W9A: $5^{\prime}$ CTT TTC CAA TCC CTC TCA TCA-3; W9S: $5^{\circ}$-CAT TGT TAG GGC CGA GGC TA-3', respectively. Primers were designed with respect to the genomic WTI sequence described in Gessler et al. (1992).

A 176-bp PCR product for exon 8 and a 218-bp PCR product for exon 9 were obtained after 35 cycles of $30 \mathrm{~s}$ at $94^{\circ} \mathrm{C}, 30 \mathrm{~s}$ at $60^{\circ}$ $\mathrm{C}$, and $1 \mathrm{~min}$ at $72^{\circ} \mathrm{C}$. PCR products were purified by centrifugation through Spin Bind DNA extraction units (FMC). One tenth of the reaction mixture was used for dideoxy-sequencing using a sequenase kit (U.S. Biochemical) with $10 \mu$ moles of the same oligonucleotides as primers according to the method of Casanova et al. (1990).

Single-strand chain polymorphism (SSCP) analysis were performed as described previously (Orita et al. 1989), and the non-denaturating gels run under two standard conditions, either at $4^{\circ} \mathrm{C}$ or at room temperature, with $10 \%$ glycerol. Primer sequences were: wt-1s, 5'-GGGCGTCCGGGTCTGAGCCT-3', and wt-1a, $5^{\prime}$-CCGGAAAAGTGGACAGTGAAGGCG- ${ }^{\prime}$ ' for exon 1 ; wt 1 2s, $5^{\circ}$-CGAGGAGCAGTGCCTGAG-3', and wt 1-2a $5^{\circ}$-GCGGAGAGTCCCTGGCGC-3', and wt-2s, 5'-CGAGAGCACCGCTGACACTG-3\% and wt-2a, 5'-GAGAAGGACTCCACTTGG'TTCCG-3' for exon 2; wt-3s, $5^{\circ}$-CCAGGCTCAGGATCTCGTGT-3', and wt-3a, 5'-AAGGACCCAGACGCAGAGC-3' for exon 3; wt-4s, 5'-TGCTTTTGAAGAAACAGTTGTG-3', and wt-4a, $5^{\prime}$-GGAAAGGCAATGGAATAGAGA-3' for exon 4; wt-5s, 5 ' GGGCTTTTCACTGGATTCTG-3', and wt-5a, 5'-CCATTTGCTTTGCCATCTCC-3' for exon 5; wt-6s, 5'-GTGAGCCACACTGAGCCTTT- $3^{\circ}$ and wt-6a, $5^{\prime}$-GGCCGGTAAGTAGGAAGAGG-3' for exon 6; wt-7s, $5^{\prime}$-GGCTTAAAGCCTCCCTTCCT$3^{\prime}$, and wt-7a, 5'-TGAGAGCCTGGAAAAGGAGC-3' for exon 7; wt-8s, $5^{\circ}$-GAGATCCCCTTTTCCAGTATCA-3', and wt-8a, 5'ACAGCTGCCAGCAATGAGAA-3' for exon 8 ; wt-9s, $5^{\prime}$-CATTGTTAGGGCCGAGGCTA-3', and wt-9a, $5^{\prime}$-CTTTTCCAATCCCTCTCATCA-3' for exon 9; wt-10s, 5'-TGTGCCTGTCTCTTTGTTGC-3' and wt-10a, $5^{\circ}$-GTTCACACACTGTGCTGCCT-3' for exon 10.

\section{Results and discussion}

The four patients were analyzed to determine the presence or absence of point mutations in WTl exons 8 or $9 . \mathrm{Ge}$ nomic DNAs isolated from peripheral blood lymphocytes 


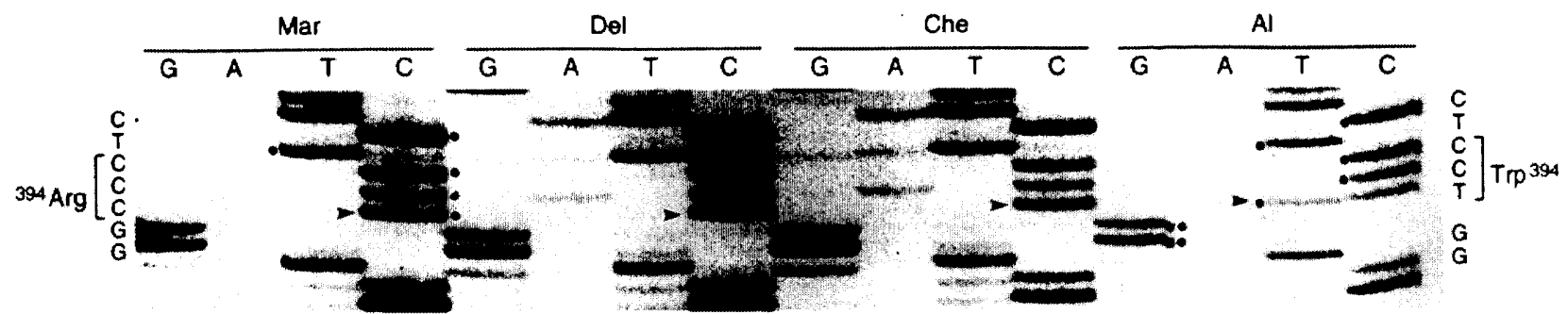

Fig. 1. Direct sequencing of PCR products from exon 9. The Denys-Drash patient $A$ /shows the $\mathrm{C} \rightarrow \mathrm{T}$ transition, a mutation not found for the Frasier individuals Che. Del and Mar. The corresponding position of the nucleotide affected by a mutation in the Denys-Drash patient $(A I)$ is marked by an arrow. $G, A, T, C$ : sequencing lanes

exon 7

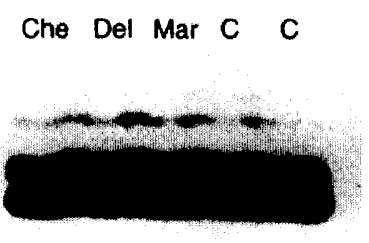

exon 10

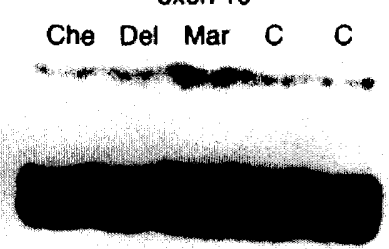

Fig. 2. SSCP analysis of WT1 exons 7 and 10 in Frasier patients. No variation was detected in the banding obtained with Che, Del or Mar when compared with normal individuals $(C)$

were subjected to direct sequencing after amplification with the appropriate primers. No mutation could be found in exon 8 in each case (data not shown). The individual with Denys-Drash syndrome displayed the same hemizygous mutation in exon 9 (Fig. 1) as the majority of patients analyzed in Pelletier et al. (1991). This C to T transition converts ${ }^{394} \mathrm{Arg}$ to $\mathrm{Trp}$ within the third zinc finger domain of WT1 and thus potentially affects the DNA-binding properties of the resulting transcription factor. On the other hand, DNA from all the patients with Frasier syndrome showed no mutations in exon 9.

However, the presence of WT1 abnormalities in Frasier syndrome cannot be excluded elsewhere in the gene, although it appears unlikely because of the absence of Wilm's tumor development. The DNAs of the three patients were tested using SSCP assay for alterations in the coding region of exons $1-10$ of WT1 with the exception of the $5^{\prime}$ half of exon 1 that is so GC-rich that it cannot be amplified reproducibly in our hands. In all reactions, there was no difference of the banding patterns compared with that seen in several control samples. An example of such an SSCP analysis is provided in Fig. 2. However, the SSCP method may fail to detect some point mutations and has been described with an estimated $80 \%$ of point mutations being detectable (Orita et al. 1990). Furthermore,

large deletions in the sex-determining region of the $\mathrm{Y}$ chromosome of such individuals or mutations localized in the sex-determining gene SRY have not been detected (Berta et al. 1992). Thus, the molecular origin of this syndrome affecting urogenital development remains an open question.

In conclusion, although the Frasier and Denys-Drash syndromes sometimes appear to be difficult to distinguish clinically, our results provide a possible molecular basis for separating these two syndromes on a genetic basis.

Acknowledgement. This work was supported by a grant from the Deutsche Forschungsgemeinschaft (Ge539/2).

\section{References}

Berta P, Morin D, Poulat F, Taviaux S, Lobaccaro JM, Sultan C. Dumas R (1992) Molecular analysis of the sex determining region from the $\mathrm{Y}$ chromosome in two patients with Frasier syndrome. Horm Res 37: 103-106

Casanova JL, Pannetier C, Jaulin C, Kourilsky P (1990) Optimal conditions for directly sequencing double-stranded PCR products with sequenase. Nucleic Acids Res 18:4028

Drash A, Sherman F, Hartmann WH, Blizzard RM (1970) A syndrome of pseudo hermaphroditism. Wilm's tumor, hypertension and degenerative renal disease. J Pediatr 76:585-593

Frasier SD, Bashore RA, Mosier HD (1964) Gonadoblastoma associated with pure gonadal dysgenesis in monozygotic twins. J Pediatr 64:740-745

Gessler M, König A, Bruns GAP (1992) The genomic organization and expression of the WT1 gene. Genomics 12:807-813

Moorthy AV, Chesney RW, Lubinsky M (1987) Chronic renal failure and XY gonadal dysgenesis: "Frasier" syndrome-A commentary on reported cases. Am J Med Genet 3:297-302

Orita M, Suzuki Y, Sekiya $\Upsilon$, Hayashi K (1989) Rapid and sensitive detection of point mutations and DNA polymorphisms using the polymerase chain reaction. Genomics 5:874-879

Orita M, Sekiya T, Hayashi K (1990) DNA sequence polymorphism in Alu repeats. Genomics 8:271-278

Pelletier J, Bruening W, Kashtan CE, Mauer SM, Manivel JC. Striegel JE, Houghton DC, Junien C, Habib R, Fouser L, Fine RN, Silverman BL, Haber DA, Housman D (1991) Germline mutations in the Wilms' tumor suppressor gene are associated with abnormal urogenital development in Denys-Drash syndrome. Cell 67:437-447 